\title{
Ethische Entscheidungen in der Politik
}

\author{
Isabelle Borucki
}

Rezension von Jung, Corinna (2012): Ethische Entscheidungen in der Politik. Die Bedeutung von Kommissionen für die politische Debatte über Patientenverfügungen. Stuttgart: Kohlhammer

Die Dissertation Jungs widmet sich in acht Kapiteln einem bislang wenig beachteten Feld: Der Rolle von Politikberatung bei der Arbeit von Kommissionen in ethisch-medizinischen Fragen am Beispiel von Patientenverfügungen. Patientenverfügungen sind aufgrund des medizinisch-technischen Fortschritts inzwischen Alltag. Deshalb befassten sich in den Jahren 2003 und 2004 zwei Kommissionen mit diesem Thema. Die Enquete-Kommission „Ethik und Recht der modernen Medizin“ des Bundestags sowie die Ad-hoc Kommission des Bundesjustizministeriums „Patientenautonomie am Lebensende“ veranschaulichen „die enorme Spannweite möglicher Positionen zwischen Lebensschutz und Patientenautonomie" (S. 13), wie die Autorin betont. Zielsetzung ihrer Untersuchung ist die Analyse des Zustandekommens unterschiedlicher Ergebnisse der beiden Kommissionen zu ethischen Themen wie der Patientenverfügung und die damit verbundene Kontingenz politischer Entscheidungsfindungen. Die Patientenverfügung stellt laut Jung ein typisches Dokument der Wissensgesellschaft als politische Begrifflichkeit dar (S. 15) und wirft die Frage auf, inwieweit wissensbasierte Politikberatungsinstrumente, bspw. Ethikexpertise, das Kräfteverhältnis von Politik und Beratung abzubilden verhelfen (S. 20, 25).

Auf Basis von 32 Experteninterviews (Mitglieder der Kommissionen, Politiker, die am 2009 verabschiedeten Gesetzentwurf zur Thematik beteiligt waren), teilnehmenden Beobachtungen an den Beratungen der Enquete-Kommission sowie Primärquellen (wie Kommissionsberichten und Bundestagsdrucksachen), analysiert die Autorin Akteurspositionierungen, Grenzziehungen, den Aufbau der Kommissionarbeit und schließlich die jeweiligen Beratungsprozesse in diesen Gremien (S. 14). Grundlegend steht so der Stellenwert von Expertise in politischen Entscheidungsprozessen zur Analyse, derer sich Jung über vier Analyseebenen nähert (S. 48): Dem Entstehungskontext (Kapitel 3), der „Betrachtung der Expertise“ (Kapitel 4), „Verwendung der Expertise“ (Kapitel 5) und schließlich „das konkrete Arbeiten in den Kommissionen“ (Kapitel 6). Die Kommissionen bilden den „empirischen Ort“ der Untersuchung (S. 42), deren Zusammensetzung und Beratungsablauf sowie Ergebnisse Jung vergleichend betrachtet. Sie analysiert in ihrem vierten Kapitel die Berufungen und professionelle Provenienz der Mitglieder und chiffriert diese nach Berufsgruppen. Die Besetzungen der Kommissionen wei- sen Gemeinsamkeiten bei der Wichtigkeit von Ärzten und Juristen als Hauptberufsgruppen hinsichtlich des Umgangs mit Wissen auf. Unterschiede findet Jung jedoch bei der Arbeitsweise und Entscheidungsfindung der beiden Gremien: Patientenverfügungen werden als „Objekte des Zusammentreffens verschiedener sozialer Welten“ (S. 133) und somit zunächst als mögliche Grenzobjekte im Sinne des Boundaryobjects Ansatzes interpretiert. Im Ergebnis zeigt sich, dass die Frage nach dem Verhältnis von Beratung und Politik bzw. die Annäherung an jene vielschichtig und komplex ist. So konstatiert Jung für ihre dritte Betrachtungsebene der Verwendung bereitgestellter Expertise von Seiten der Politik einen „sich über mehrere Ebenen erstreckende[n] Beratungsbedarf, Beratungen zu Beratungen“ als „keine Seltenheit“ (S. 159). Insgesamt haben „die Sachverständigen große und nicht zu unterschätzende Einflussmöglichkeiten " auf Entscheidungsprozesse in Kommissionen. Beim tatsächlichen Zustandekommen der Entscheidungen, der vierten Betrachtungsebene, beobachtet Jung interne wie externe Mikropolitik (S. 161) und verknüpft diese mit dem Boundary-objects Ansatz. Dieses Theorem verdeutlicht, dass in der Enquete nicht die Patientenverfügung als Grenzobjekt fungiert, sondern die Enquete selbst. Es zeigt sich also eine deutliche Kontextgebundenheit der Aushandlungen.

Insgesamt zeichnet sich die Studie durch verständliche, zumeist klare Argumentation und Sprache sowie ausgezeichnete Leserführung aus. Sie wird von einer transparent gemachten methodischen Offenlegung im Anhang sowie ein Namensund Sachregister komplettiert. Allenfalls das Literaturverzeichnis hätte zur besseren Auffindbarkeit der Primärquellen diese gesondert aufführen können. Jungs qualitativ fokussierte Studie, die viel unterschiedliches Material zusammengetragen hat, dichte Beschreibungen und Typisierungen in verständlicher Weise präsentiert, ist theoretisch dem Sozialkonstruktivismus zuzuordnen. Diesbezüglich wäre eine deutlichere und umfangreicher ausgeführte theoretische Basierung durchaus interessant gewesen (z. B. Fußnote 3, S. 75). Der Anspruch, eine Theorie aus den Daten selbst heraus zu entwickeln (S. 37-38), ist lobenswert, es wird allerdings in der Analyse und Interpretation der Daten nicht deutlich, was genau nun die emergierende Theorie ist, was diese ausmacht und wie sie in den Theoriekanon der Soziologie allgemein und der Politikberatung und Medizinethik speziell einzuordnen ist.

Isabelle Borucki

Universität Trier

isabelle.borucki@uni-trier.de 